\title{
A Data-driven Algorithm for Online Detection of Components and System Faults in Modern Wind Turbines at Different Operating Zones
}

\author{
Azzeddine Bakdi* ${ }^{1}$, Abdelmalek Kouadri², Saad Mekhilef ${ }^{3}$ \\ 1: Department of Mathematics, University of Oslo, 0851 Oslo, Norway. \\ 2: Signals and Systems Laboratory, Institute of Electrical and Electronics Engineering, University \\ M’Hamed Bougara of Boumerdes, 35000 Boumerdès, Algeria.
}

3: Power Electronics and Renewable Energy Research Laboratory (PEARL), University of Malaya, 50603 Kuala Lumpur, Malaysia.

bkdaznsun@gmail.com, ab kouadri@hotmail.com, $\underline{\text { saad@um.edu.my }}$

\begin{abstract}
:
Advanced Fault Detection (FD), diagnosis, and isolation schemes are necessary to realize the required levels of reliability and availability and to minimize financial losses against failures, explicitly in modern Wind Turbine Systems (WTSs) which are designed to generate electrical energy as efficiently and reliably as possible. This paper presents the design of a novel and inclusive FD framework on the basis of data-driven methods, it is intended for the online early detection of involuntary anomalies of various types and locations. Conventional methods are based on the exact model and/or signal patterns or hardware redundancy, and generally fail to address this issue. On the contrary, the presented algorithm is motivated by the availability of fast sensors and powerful computers yielding big data which can be explored to extract and exploit useful information. In a typical WTS, FD procedures face particular challenges attributed to high levels of measurement noise and sparse changes due to the fast dynamics as well as the switching control strategy along with transient phases. In this scope, a minimum and informative set of measured variables is proposed to accurately and completely describe the system behavior under its entire operation range; datasets are hence defined for appropriate dimensions and sampling time to satisfactorily model the measured data patterns and variability. Among data-based strategies, multivariate and univariate statistical analysis tools are recommended for this approach. A method based on Principal Component Analysis (PCA) is used in this study for its capabilities of dimensionality reduction, information de-correlation, and noise rejection. Multi-PCA-models are consequently used to train parallel statistical models through the proposed set of fault-relevant variables and used for online FD. Moreover, this method is reinforced by an adaptive threshold scheme based on an efficient modified EWMA control chart, the overall algorithm is robust to outliers and highly sensitive to small abnormalities and abrupt changes. Static and dynamic applications are investigated for modern wind turbine systems under different operating zones, and faults having different severity levels are studied ranging from sensors to actuators and up to system faults. The overall constructed algorithm shows significant potential applications compared to several methods recently reported in the literature in terms of applicability potential, robustness, and detection sensitivity.
\end{abstract}

\section{Introduction}

Beside clean energy contribution, wind energy comprises a nonfinite renewable energy source and it is the most competitive source of new power generation; In 2016, the wind was the largest destination for power sector investments [1]. Wind energy already meets $10.4 \%$ of the EU's power demand with high penetration levels in several countries (Denmark 42\%; Spain 20\%; Germany 13\%; and UK 11\%) [1]. In this direction, contemporary wind turbine size has increased up to more than 200 meters. The rated power of wind turbines was in the order of less than $1000 \mathrm{~kW}$ during the mid-1990s, while currently, the market of modern wind turbines is dominated by multi-megawatt machines. Nowadays, these are elevated to machines of $10 \mathrm{MW}$ rated power, destined mainly to serve offshore wind energy applications. Moreover, machine capacity is expected to increase even more through future innovative concepts [2].

A wind turbine system (WTS) usually operates continuously (24/7) under increasing challenges to satisfy high power demands matching the desired power quality. With its increased size, complex interactions, and numerous components operating at high power, a WTS is frequently subject to components as well as system failures at critical 
rates [3, 4], as illustrated in Fig. 1 and Fig. 2, causing long downtime reparation periods up to several days in addition to huge financial losses due to its respective size. Fault Detection (FD) tasks hence play a crucial role in the production capacity as well as quality achieved by wind turbines and their financial outcomes. Poor monitoring system directly affects: (i) the outcome of a system through increased operational and maintenance costs [4, 7], and (ii) reduced availability to generate power as a result of turbine downtime. The two conditions certainly encourage WTS operators to employ the most advanced and adapted monitoring methods to reduce these failure rates and time to repair and to maximize the WTS output quality and performance.

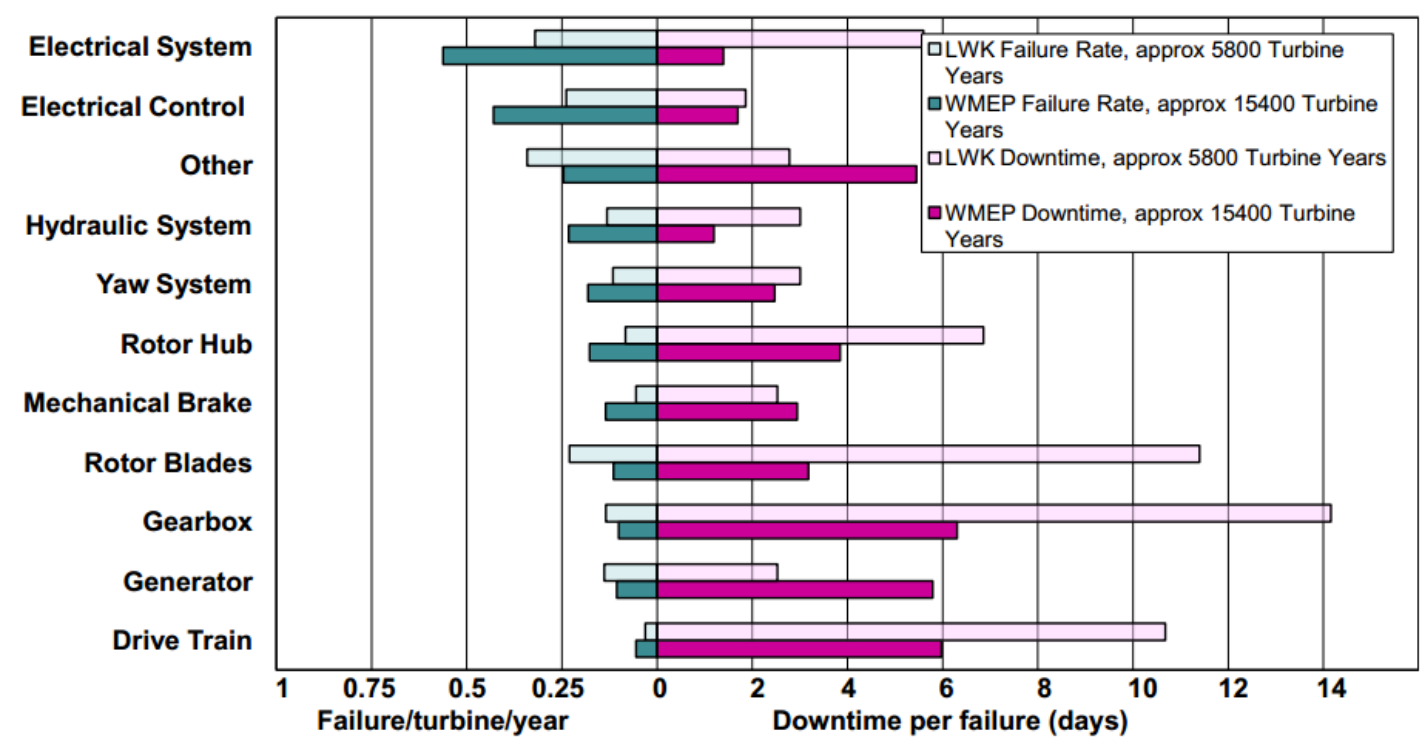

Fig 1. Failure rates and downtime per failure of onshore European wind turbines [3].

Accordingly, and for the sake of achieving resilient wind energy systems, extensive theoretical and experimental research have considered the problem of early detection of distinct WTS faults and abnormalities focusing mainly on the critical components. Authors in [8] present a comprehensive survey about fault ride through techniques for WTSs with proposed controllers, some techniques are also considered by [9] focusing on the WTS doubly-fed induction generator. Fault analysis and load monitoring are also considered in [10] and [11] respectively. Qualitatively, WTS health monitoring is considered in [12 - 15]; Authors in [12] considered the biocomposite material selection in a specific weather conditions and the impact on structural health and recommended the use of biocomposite green material instead of synthetic fiber; long-term operation and structural health related faults in WTS were addressed in [13] including blades [15], gearbox, bearing, and mechanical braking; Authors in [14] proposed the use of statistical pattern recognition for WTS health monitoring based on signals from ultrasonic/ acoustic emission, thermal imaging, fatigue and strain. Testing, inspection and monitoring technologies for WTS components such as turbine blades, rotor bearing conditions, and converter are considered separately in [16 - 18], respectively. A survey is provided in [16] on mechanical/ fatigue/ modal testing as well as inspecting and monitoring technologies; the authors arrived to the fact that full-scale testing must be performed. Bearing condition monitoring in WTSs was considered in [17] through offline acoustic/ temperature/ vibration monitoring. The diagnosis of converter faults was considered in a survey in [18], where common reasons of converter faults were listed with their mostly used diagnosis tools. In reality, all unit operations of a WTS are interconnected and their variables are strongly coupled. Since the occurrence of a particular anomaly generally affects all the remaining units, the global structure of the system must be considered for detecting possible anomalies of different types and low impacts in their very early stages.
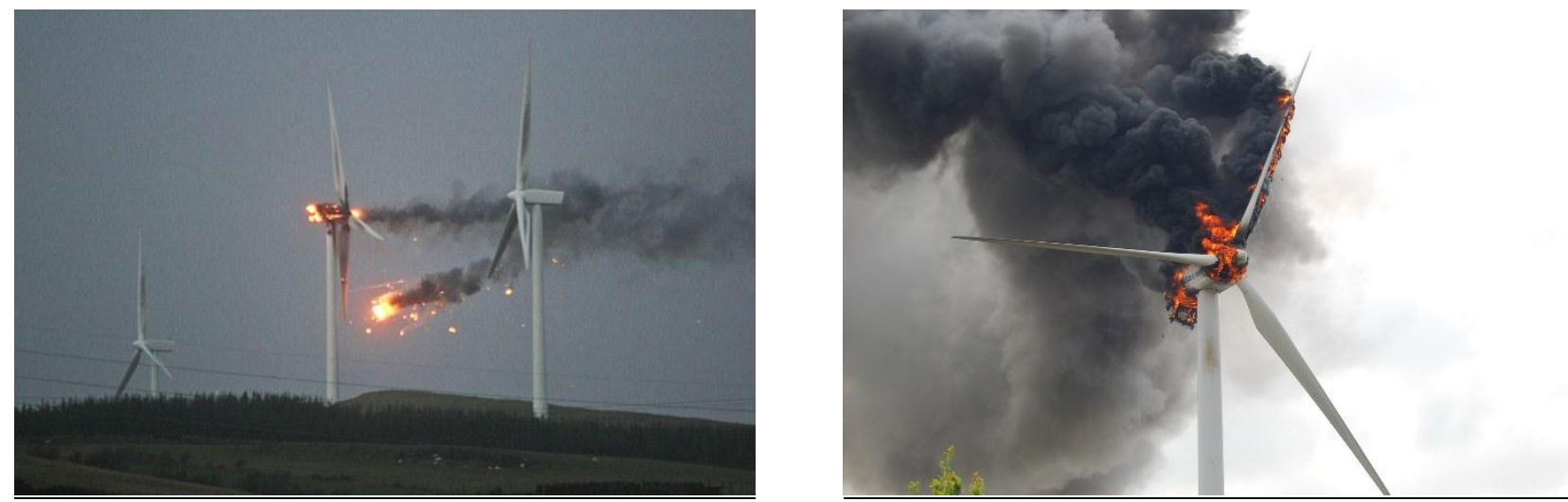

Fig 2. Several types of WTS faults, if undetected, may lead to severe damages and power losses [5, 6]. 
Data-driven frameworks are proposed in this paper for global monitoring of modern WTSs and early detection of involuntary faults of different types, impacts, and locations. Traditional FD methods in WTSs are model-based, signalbased, and some techniques based on hardware redundancy. Model-based methods [19 - 21] include the use of analytical relations [19] and state observers or parameter estimators assuming the structure is given and the system is observable; Set-membership approaches are used in [20] with Bayesian reasoning for FD in WTSs, this method assumes bounded errors and bounded noise levels with known distributions; in a quite similar way, fuzzy membership functions are used in [21] to construct a Takagi-Sugeno fuzzy model for a WTS for FD purposes. Model-based techniques often rely on a complete and exact analytical/ statistical/ AI model which cannot be accurately obtained for modern WTSs which are complex and large in size. Signal-based FD techniques generally involve Kalman/ $H_{\infty}$ filtering and observers [22], or fuzzy identifications [23]; these techniques require prior known signals with their models and patterns and they are used for local monitoring such as drive-train and converter. In the same direction, the major drawback for hardware redundancy strategies is the increased cost, maintenance efforts, complexity, and failure probabilities caused by installing extra sensing devices. Nowadays, with fast computing and sensing technologies having the ability to acquire and process big datasets, data-based methods are receiving an increasing attention for many applications in complex systems such as WTSs control and monitoring [24, 25]. In general applications, multivariate statistical data-driven methods make a superior alternative solution for semi-supervised FD, these methods include Principal Component Analysis (PCA) [26], Independent Component Analysis (ICA) [27], Partial Least Squares (PLS) [28], Higher-Order Cumulants Analysis (HCA) [29], Fisher Discriminant Analysis (FDA) [30], and Slow Feature Analysis (SFA) [31]. On the basis of large size multivariate data of low statistical rank, these methods are very efficient in extracting few independent sources carrying most of the information and describing dominant variation in the overall system.

The use of multivariate statistics for big data analysis aiming at detecting wide range of anomalies in WTSs has not been reported so far. The aforementioned model-based [19 - 21] and signal-processing-based [22, 23] techniques are hard to be designed and generalized for different types of WTSs in real-time applications. Related works dealing using data-based FD techniques in WTSs include Monte-Carlo analysis [24], Gibbs sampling algorithm [25], and support vector machines (SVM) [37, 38] classification; These approaches, however, appear to be fully supervised since they are trained through extra labeled data with sufficient measurements collected during faulty operations. Unfortunately, such data cannot be obtained in practice. Another drawback of these approaches can be seen through the high dependability on the training datasets which consequently narrows their application scope, since SVM is limited to classifying future scenarios similar to training data. These classifiers are also highly sensitive to noise, authors in [37, 38] proposed the use of low pass filters for signal de-noising. However, such filtering destroys some of the signals details and therefore delays the detection and reduces the sensitivity against small deviations.

PCA and other multivariate statistical analysis techniques are commonly used for monitoring processes which are generally stationary and operate under deterministic conditions, fixed set-points, and slow dynamics. On the contrary, several challenges arise in the scope of FD in WTSs, which have particular types of signals and data patterns; (i) The high levels of measurement signal-to-noise-ratio (SNR) cause many outliers that mislead the detection algorithm causing many false alarms and/or mask some faults leading to missed detections; (ii) The switching control strategy of WTSs cause another difficulty making the system highly nonlinear and operating under continuously changing modes; (iii) Fast dynamics of such a system hence often cause frequent transient stages which also contributes to the difficulty of extracting the statistical information and estimating the monitoring parameters. In this work, a minimum and informative set of fault-relevant variables is defined to construct a reliable FD scheme through multivariate statistical analysis. The appropriate datasets are hence spanned through this variables with adequate dimensions and sampling intervals to properly model the data and extract the statistical parameters.

PCA $[32,33]$ is mainly used in this work to efficiently construct parallel (multi) statistical models for the WTS under different operation zones. In addition, Dynamic PCA (DPCA) method [34 - 36] is used to capture serial correlation and consider the dynamics of the WTS which operates under varying dynamic loads due to environmental conditions. Data-driven and particularly PCA methods were proved powerful in detecting anomalies. These methods, however, rely on basic assumptions such as data normality and stationarity, which is not the case for WTSs. The continuously evolving operation during normal conditions raise a major challenge of discriminating changes due to anomalies from those normal evolutions. Furthermore, WTSs operate in a switching manner under multi-modes which are further continuous and joint. Hence, learning a global statistical model results in a biased and poor representation for the system functionality; on the other hand, constructing discrete models for the variability of such big data is computationally heavy and not feasible to the objective of fast detection. In this direction, multi-model PCA-based strategy is proposed for 
efficient and accurate modeling of the covariance structures under both operating zones. From a data analysis point of view, sparse changes due to switching control and transients in addition to the high SNR toughen both tasks of statistical modelling and analysis. Even under a distinct set-pint, the noise-decoupling aspect of PCA is not well achieved, both principal and residual subspaces defined by PCA include noise features in addition to main features describing natural variability. Under such imperfect decomposition, discriminating sparse changes from noise-related outliers is inflexible in both subspaces. To cope with data uncertainties and their impact on training and statistical analysis and evaluation, the algorithm is reinforced with an adaptive threshold scheme to increase the robustness and FD sensitivity and maintain a stable monitoring performance over long operation periods.

On the basis of maximum information extraction and appropriate statistical analysis and evaluation, the developed algorithm can be generalized to any WTS which exhibits increased challenges inherited from any electromechanical system including nonlinear and complex interactions with dynamic behavior. Due to systems large scale, faults can reside at any level across its numerous units, leading to electrical or mechanical abnormalities. These faults also have different severity levels and impacts on the system and exhibit different behaviors to be detected. Fqults in sensors generally lead to wrong measurements and system reconfiguration in feedback closed loops, and thus they have a low influence on the WTS performance making them harder to be detected. However, faults in actuators have a higher severity on the system performance and safety such as slow control actions for pitching the blades and nonoptimal power generation, this definitely imposes instantaneous or fast detection. System faults including drive train vibrations also have a remarkable severity with increased detection challenges. Under these circumstances, the objective of the presented algorithm is the early detection of several types of faults according to their given constraints and severity levels. This certainly provides an invaluable warning time to implement the appropriate counteractions and prevent fault evolution and system failure, and therefore increases the WTS lifetime as well as reliability and reduces maintenance costs.

The rest of this paper is organized as follows; Section 2 provides a detailed description of the continuous and joint operating modes of a typical WTS according to its overall control strategy, and main signals used for monitoring application as well as all considered sensor, actuator, and system faults. Section 3 brings forward different stages of the developed FD algorithm considering PCA based statistical learning for static and dynamic modelling followed by a developed adaptive threshold scheme for statistical evaluation. FD application on the WTS is described in Section 4 and drawn results are discussed. Finally, major findings and potential applications are given as a general conclusion in Section 5.

\section{Wind turbine}

\subsection{Wind turbine system description}

A horizontal-axis variable-speed wind turbine with a rated power of $P_{r}=4.8 \mathrm{MW}$ is considered in this work. This system has three blades and it consists of four main unit operations as indicated in fig. 3 [37]: blade and pitch system (hydraulic), drive train, generator and converter, and controller. The drive train is used to increase the rotational speed from the rotor $\omega_{r}$ to the generator $\omega_{g}$, and the full converter is coupled to the generator which converts the mechanical wind energy applied on the blades to electrical energy. Based on the wind speed $v_{w}$, the aerodynamics of the turbine are controlled for optimal power generation $P_{g}$. The system controls the three pitch systems $\left(\beta_{1}, \beta_{2}, \beta_{3}\right)$ and the convertor to allow respectively pitching the blades and setting the generator torque $\tau_{g}$ to control the rotational speed of the generator and the rotor. The overall system is nonlinear with switching control structure and the measurements are noisy.

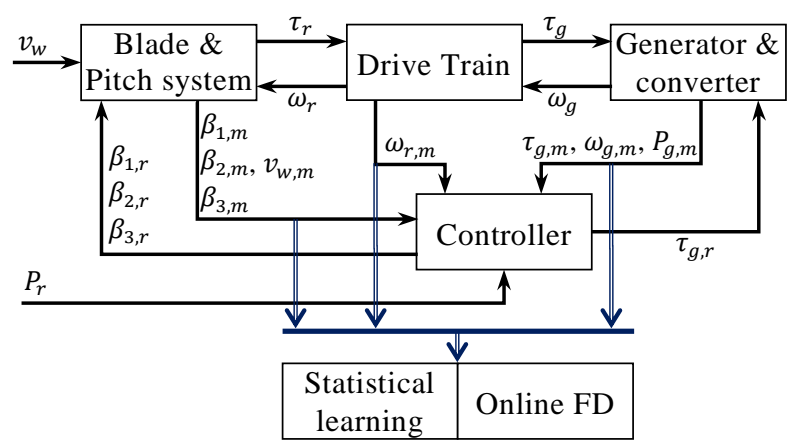

Fig 3. Simplified schematic diagram of the wind turbine system illustrating main unit operations and relevant signals $[38]$. 
Due to increased academic and industrial interests in control design, performance analysis, and monitoring of modern wind turbines, a generic and realistic benchmark was proposed and developed [37, 38] for monitoring studies and comparisons of results. Based on the Benchmark model and faults, an international competition on fault detection and isolation in wind turbines was released in 2010 by kk-electronic with MathWorks and Aalborg University. This benchmark was also used for several research applications [39 - 41]. It allows simulating the wind turbine system under power production operation modes (based on the mean wind speed), namely zone II and zone III which are indicated in fig. 4. Zone II corresponds to optimal power generation (or partial load) during insufficient wind speed, and Zone III represents constant power generation. The wind turbine is at standstill for very low wind speed (bellow cut-in level), and it is pitched out of the wind stopping the rotation and the energy production at very high wind speed (above cut-out level). These two modes are excluded from the benchmark model and no monitoring task is required. The operational zones are considered for data acquisition, statistical modelling, and monitoring, as will be presented in sections 3 and 4 .

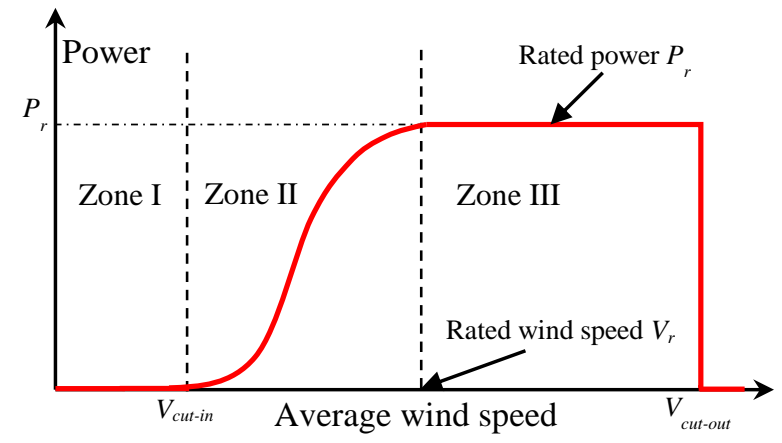

Fig 4. Wind turbine operation zones.

Table 1. Process variables for the wind turbine system.

\begin{tabular}{llc}
\hline Variable & Description & Unit \\
\hline$v_{w}, v_{w, m}$ & Real wind speed acting on the turbine blades and its measured value, & $\mathrm{m} / \mathrm{s}$ \\
& respectively. & $\mathrm{N} \mathrm{m}$ \\
$\tau_{r}, \tau_{g}$ & Real rotor and generator torques, respectively. & $\mathrm{m} \mathrm{m}$ \\
$\tau_{g, r}, \tau_{g, m}$ & Generator reference and measured torques, respectively. & $\mathrm{rad} / \mathrm{s}$ \\
$\omega_{r}, \omega_{g}$ & Real rotor and generator rotational speed. & $\mathrm{rad} / \mathrm{s}$ \\
$\omega_{r, m j}, \omega_{g, m j}$ & Measured rotational speed of the rotor and the generator, respectively. Two & $\mathrm{W}$ \\
$P_{r}, P_{g, m}$ & measurements for each one. $j=1,2$. & $\circ$ \\
$\beta_{i, r}$ & Rated and measured generated power, respectively. & $\circ$ \\
$\beta_{i, m j}$ & Reference to the pitch position (three blades). $i=1,2,3$. & \\
& Measured pitch position (three blades, two measurements for each one). $i=$ & \\
\hline
\end{tabular}

A real acquired sequence of varying wind speed with significant measurement noises is used to study the system under both operation zones. The system is equipped with duplicated sensors to measure the three pitch positions $\left(\beta_{i, m j}, i=1,2,3\right.$, and $\left.j=1,2\right)$ as well as the speeds of the generator and rotor $\omega_{g, m j}$ and $\omega_{r, m j}(j=1,2)$, respectively. Measured and reference system variables are also described in Table 1. Random measurement noise is considered in this work, it is associated with all measurement sensors, and it is initialized through a random seed generator to ensure randomness and independence of data acquisition. A total of ten sensors are therefore used and they are all subject to two kinds of faults: fixed value and gain factor as listed in Table 2 [37].

Table 2. Sensor, actuator, and system benchmark faults in the wind turbine process [37].

\begin{tabular}{|c|c|c|c|c|c|}
\hline Fault & & Type & Description & Severity & DTC \\
\hline & $\begin{array}{l}\text { 1a) } \\
\text { 1b) }\end{array}$ & $\begin{array}{l}\text { Fixed value } \\
\text { Gain factor }\end{array}$ & $\begin{array}{l}\text { False measurements in blade positions, } \\
\text { reconfigure system }\end{array}$ & & \\
\hline $\begin{array}{l}\text { Sensors } \\
\text { Faults }\end{array}$ & $\begin{array}{l}2 a) \\
\text { 2b) } \\
3 a)\end{array}$ & $\begin{array}{l}\text { Fixed value } \\
\text { Gain factor } \\
\text { Fixed value }\end{array}$ & $\begin{array}{l}\text { False measurements in rotor speed, } \\
\text { reconfigure system } \\
\text { False measurements in generator speed, }\end{array}$ & Low & $<10 T_{S}$ \\
\hline
\end{tabular}




\begin{tabular}{|c|c|c|c|c|c|}
\hline & 3b) & Gain factor & reconfigure system & & \\
\hline \multirow{3}{*}{$\begin{array}{l}\text { Actuators } \\
\text { faults }\end{array}$} & 4a) & Offset & $\begin{array}{l}\text { Bad torque control, none optimal power } \\
\text { generation }\end{array}$ & Medium & $<5 T_{s}$ \\
\hline & 5a) & $\begin{array}{l}\text { Changed } \\
\text { dynamics }\end{array}$ & $\begin{array}{l}\text { Slow control actions due to problems with } \\
\text { pump or leakage }\end{array}$ & High & $<8 T_{s}$ \\
\hline & $5 b)$ & $\begin{array}{l}\text { Changed } \\
\text { dynamics }\end{array}$ & Slow control actions due to air in oil & Medium & $<600 T_{S}$ \\
\hline $\begin{array}{l}\text { System } \\
\text { fault }\end{array}$ & 6) & $\begin{array}{l}\text { Changed } \\
\text { dynamics }\end{array}$ & Increased level of drive train vibration & Medium & free \\
\hline
\end{tabular}

DTC: Detection time delay condition, where $T_{s}=10 \mathrm{~ms}$ s the sampling time.

The controller operates according to the average wind speed, it generates the three reference angular positions $\beta_{i, r}, i=1,2,3$, and the reference generator torque $\tau_{g, r}$. The hydraulic pitch system is modelled by a second-order transfer function, specifying the relationship between the real pitch angles $\beta_{i}$ and their reference values $\beta_{i, r},(i=1,2,3)$ :

$$
\frac{\beta_{i}(s)}{\beta_{i, r}(s)}=\frac{\omega_{n}{ }^{2}}{s^{2}+2 \xi \omega_{n} s+\omega_{n}{ }^{2}}
$$

With $\left[\omega_{n}, \xi\right]=[11.11,0.6]$ during normal operation (normal response of the actuator to the controller reference value).

The converter dynamics on the other side are modeled by a first-order transfer function, relating the real generator torque $\tau_{g}$ to its reference value (generated by the controller) $\tau_{g, r}$ :

$$
\frac{\tau_{g}(s)}{\tau_{g, r}(s)}=\frac{1}{\tau s+1}
$$

With $\tau=20 \mathrm{~ms}$.

\subsection{Benchmark faults}

Different types of faults are studied within this WTS, including sensors, actuators, and system faults. Actuator faults 4a), 5a), and 5b) as described in Table 2, are simulated with a constant offset of the generator torque to emulate bad torque control as well as changing the dynamics $\omega_{n}$ and $\xi$ for the case of slow control actions due to pump problems such as leakage and air in oil.

Since the system is operating under feedback (closed loops) control and duplicated measurement system is adopted through physical redundancy, sensors faults have a lower severity with a relatively larger Detection Time Constraint (DTC) as indicated in Table 2. Yet, they must be handled within this limited DTC before becoming critical. On the other hands, actuator faults have a medium to high severity on the system's performance and safety since these controllers are expected to execute the main control actions governing the overall process and its performance. Thus, abrupt controller faults must be detected within a smaller time period. Lastly, increased level of vibration in drive train unit with long-term operation will lead to system wearing and reduced lifetime, thus, this fault has no detection time constraint, but it causing a medium severity on the system in terms of downtime reparation and maintenance costs.

\section{Constructed WTS FD Algorithm}

Monitoring and FD algorithms are effectively designed through an appropriate set of fault-relevant variables that should be selected. Minimum set is required since the redundancy of sensory devices is usually avoided due to cost and complexity. It is also vital to limit the monitoring variables to those signals with relevant information on the system behavior and capable of reflecting faults symptoms. Out of this set, additional variables may unnecessarily increase the time and computation complexity of the algorithm and even worsen the detection performance. On the other hands, describing the system functionality requires a complete set of informative signals. The best dataset must be acquired and used for statistical modelling, information extraction, and abnormalities detection. Among 23 WTS signals indicated in section 2 and listed in Table 1, only measured variables are used for FD in the developed approach, including these 13 variables: $v_{w, m}, \omega_{r, m 1}, \omega_{r, m 2}, \omega_{g, m 1}, \omega_{g, m 2}, \tau_{g, m}, P_{g, m}, \beta_{1, m 1}, \beta_{1, m 2}, \beta_{2, m 1}, \beta_{2, m 2}, \beta_{3, m 1}$, and $\beta_{3, m 2}$.

\subsection{PCA/ DPCA-based FD}

This set of relevant monitoring signals, acquired at a fixed sampling time, form a big data matrix $\boldsymbol{X}_{\mathbf{0}} \in R^{n \times m}$ of $n$ samples and $m$ WTS variables. The well-known optimal dimensionality reduction technique PCA is used to transform 
the original large-size multivariate data from a correlated high dimensional space into a lower dimensional space. This space has an orthonormal basis of uncorrelated components capturing most of the variation as present in the original data.

In order to extract information from variables having different units and different trends, the original signals are first auto-scaled to have zero-mean and unity-variance so to avoid their weighting and bias, this results in an auto-scaled data matrix $\boldsymbol{X} \in R^{n \times m}$ of the same dimension. Through singular value decomposition, the amounts and orthogonal directions of variation in this data are obtained. PCA is often applied on the covariance matrix $\boldsymbol{S}$ representing the covariance structure among the WTS signals.

$$
S=\frac{1}{n-1} X^{T} X=P \Lambda P^{T}
$$

This structure is decomposed into $\boldsymbol{P}$ and $\boldsymbol{\Lambda}$ matrices where: $\boldsymbol{P} \in \boldsymbol{R}^{m \times m}$ is the loadings matrix and it contains the $m$ orthonormal loadings (eigenvectors), and $\boldsymbol{\Lambda} \in \boldsymbol{R}^{m \times m}$ is the diagonal descending-order eigenvalues matrix. The loadings represent the directions of variation in the auto-scaled signals, and their associated eigenvalues represent the variance as the amount of variation per each particular direction. PCA dimensionality reduction is therefore achieved by defining a barrier between main useful information which is retained to represent the statistical WTS model and the rejected information representing noise. Among $m$ loadings, also called components, only $l<<m$ of the first significant Principal Components (PCs) having large dominant eigenvalues are retained. And thus explain the highest variances. Several techniques are generally used to determine $l$ such as: Kaiser-Guttman method by retaining only those components whose eigenvalues are greater than the average of all components, which is one in the case of auto-scaled data; And Cumulative Percent Variance method which is commonly used by retaining minimum $l$ components having their sum of variances greater than a preselected Percentage Variance $(P V)$.

The loadings and diagonal eigenvalues matrices $\boldsymbol{P}$ and $\boldsymbol{\Lambda}$ are respectively decomposed, and define the two PCA subspaces according to the number of principle components $l$ :

$$
\left\{\begin{array}{l}
\boldsymbol{P}=[\widehat{P} \mid \widetilde{P}] \\
\Lambda=\left[\begin{array}{ll}
\widehat{\Lambda} & 0 \\
0 & \widetilde{\Lambda}
\end{array}\right]
\end{array}\right.
$$

where $\widehat{\boldsymbol{P}} \in \boldsymbol{R}^{m \times l}$ and $\widetilde{\boldsymbol{P}} \in \boldsymbol{R}^{m \times(m-l)}$ are respectively the projection operators onto the principal and residual subspaces defined by the modeled and non-modeled variations in the WTS. In the same manner, $\widehat{\boldsymbol{\Lambda}} \in \boldsymbol{R}^{l \times l}$ and $\widetilde{\boldsymbol{\Lambda}} \in \boldsymbol{R}^{(m-l) \times(m-l)}$ are the diagonal matrices describing retained and ignored variances, respectively.

In order to model the WTS dynamics and consider the autocorrelation among its measured variables, Dynamic PCA (DPCA) method is used. Every single variable is augmented by adding time-lagged values and thereby expanding the data matrix, resulting in a data matrix with $\lg$ time shifted lags which generally depend on the dynamics order and given an appropriate sampling time. One of the common methods used to determine the lag structure was given in [35] where linear correlations are treated as linear relationships (LRs). This method is based on the number of LRs within an extended covariance matrix, this number is generally deduced from the matrix rank deficiency. Due to the presence of noise in data, this method suggests the use of the number of PCs as the matrix rank, and therefore the approximate nullity of the matrix can be determine and considered as the number of LRs. This method recursively adds one more lag to all variables in the original data matrix as long as it yields new extra LRs. According to [35], the resulting number of lags for this method is usually 1 or 2 , depending on the order of the system dynamics, and it could be higher to offer a better linear approximation of the nonlinear correlations in case of highly nonlinear systems.

The augmented data matrix $\boldsymbol{X}_{\boldsymbol{d}} \in R^{n \times m}$ consists of $n$ observations and $m=(\lg +1) \times$ number of variables, and each row vector is given at $k^{\text {th }}$ instance as:

$$
\boldsymbol{X}_{\boldsymbol{d}}(k)=\left[\boldsymbol{X}_{\mathbf{0}}(k), \boldsymbol{X}_{\mathbf{0}}(k-1), \ldots, \boldsymbol{X}_{\mathbf{0}}(k-\lg )\right]
$$

where $\boldsymbol{X} .(k)$ is a row vector representing one measurement of all variables at sample $k$. Note in this stage that the second dimension of the augmented data matrix is multiples of the number of WTS variables. The augmented data matrix $\boldsymbol{X}_{\boldsymbol{d}}$ is also auto-scaled and decomposed according to the previous equations (3) and (4), resulting in a dynamic statistical model for the WTS.

In either static or dynamic case, when a sample measurement from the WTS (one raw vector) $\boldsymbol{x} \in \boldsymbol{R}^{1 \times m}$ is acquired, it can be decomposed into:

$$
x=x \widehat{\boldsymbol{P}} \widehat{\boldsymbol{P}}^{\mathrm{T}}+x \widetilde{\boldsymbol{P}} \widetilde{\boldsymbol{P}}^{\mathrm{T}}=x \boldsymbol{C}+x(I-C)=\widehat{x}+e
$$


where $\widehat{\boldsymbol{x}}$ and $\boldsymbol{e}$ are referred to respectively as the projection of $\boldsymbol{x}$ onto the principal and residual subspaces. Consequently, $Q$ and $T^{2}$ statistics measure the variability of this measurement within the residual and the principal subspaces, respectively.

The Squared Prediction Error (SPE) or $Q$ statistic is defined as:

$$
Q=x(I-C)(I-C)^{\mathrm{T}} \boldsymbol{x}^{\mathrm{T}}=\|\boldsymbol{e}\|^{2}
$$

and its upper control limit $Q_{\alpha}$ is given as a fixed threshold at a given significance level $\alpha$ as:

$$
\left\{\begin{array}{l}
Q_{\alpha}=\theta_{1}\left[\frac{z_{\alpha} h_{0} \sqrt{2 \theta_{2}}}{\theta_{1}}+1+\frac{h_{0} \theta_{2}\left(h_{0}-1\right)}{\theta_{1}{ }^{2}}\right]^{\frac{1}{h_{0}}} \\
\theta_{i}=\sum_{j=l+1}^{j=m} \lambda_{j}{ }^{i}, i=1,2,3 \text { and } h_{0}=1-\frac{2 \theta_{1} \theta_{3}}{3 \theta_{2}{ }^{2}}
\end{array}\right.
$$

where $z_{\alpha}$ is the critical value for an appropriately chosen confidence level $(1-\alpha)$ of the standard normal distribution.

While the Hotelling $T^{2}$ statistic is defined as:

$$
T^{2}=\boldsymbol{x} \widehat{\boldsymbol{P}} \widehat{\boldsymbol{\Lambda}}^{-1} \widehat{\boldsymbol{P}}^{\mathrm{T}} \boldsymbol{x}^{\mathrm{T}}
$$

with a fixed upper control limit $T_{\alpha}$ defined for a significance level $\alpha$ as:

$$
T_{\alpha}=\frac{\left(n^{2}-1\right) l}{n(n-l)} F_{\alpha}(l, n-l)
$$

where $F_{\alpha}(l, n-l)$ is the critical value at the confidence level $(1-\alpha)$ of the Fisher-Snedecor distribution with $l$ and $(n-$ l) degrees of freedom.

At this stage, monitoring the system functionality is reduced to monitoring the two resulting statistics. The WTS is considered faulty whenever the variability from each of the two subspaces is too large, in other words, when one of the two statistics exceeds its associated control limit, i.e. $Q>Q_{\alpha}$ or $T^{2}>T_{\alpha}$.

\subsection{Improved FD Algorithm through adaptive threshold}

For adaptive FD in the WTS, we further propose to reinforce SPCA and DPCA methods with an adaptive threshold scheme having the following advantages [32, 33]: (i) stable monitoring performance over long operation periods by dynamically updating the control limits to new appropriate values; (ii) wide range of values as control limits to accurately measure the deviation and increase fault detection sensitivity of different types of wind turbine faults while significantly increasing robustness to false alarms; (iii) exploit the use of historical statistics to properly evaluate the system deviation from previous normal operation and ensure a reliable and effective WTS FD algorithm.

Adaptive thresholds are developed for the observed sequence statistics $Q=\left[q_{1}, q_{2}, \ldots, q_{k}\right]$ and $T^{2}=$ $\left[t_{1}, t_{2}, \ldots, t_{k}\right]$. Instantaneous thresholds for these statistics are developed on the basis of Exponentially Weighted Moving Average (EWMA) control chart. In its conventional version, the EWMA $Y_{\lambda}[k]$ at time sample $k$ of the observed value $Y[k]$ is given as:

$$
Y_{\lambda}[k]=\lambda Y[k]+(1-\lambda) Y_{\lambda}[k-1]
$$

where $\lambda$ is a smoothing constant between 0 and 1 . However, a modified EWMA version with a limited window length is used to develop dynamic control limits for FD in the WTS:

$$
Y_{c}^{w}[k]=\frac{\sum_{i=1}^{w} c^{i} Y[k-w+i]}{\sum_{i=1}^{w} c^{i}}
$$

wherew is the window length and $c>1$ is the exponential weighting factor. The instantaneous value at $k^{\text {th }}$ sample of adaptive threshold $Q_{A d}[k]$ for the $Q$ statistic is given as:

$$
Q_{A d}[k]=\max \left\{\frac{1}{c_{q}^{w_{q}}}\left(Q_{\alpha} \sum_{i=1}^{w_{q}} c_{q}^{i}-\sum_{i=1}^{w_{q}-1} c_{q}^{i} q_{k-w_{q}+i}\right), 0.2 Q_{\alpha}\right\}
$$

with the following associated condition:

$$
q_{k-w_{q}+i}<Q_{A d}\left[k-w_{q}+i\right] \text { for } i=1,2, \ldots, w_{q}-1
$$


And in the same manner, The instantaneous value at $k^{\text {th }}$ sample of adaptive threshold $T^{2}{ }_{A d}$ for the $T^{2}$ statistic is given as:

$$
T^{2}{ }_{A d}[k]=\max \left\{\frac{1}{c_{t}^{w_{t}}}\left(T_{\alpha} \sum_{i=1}^{w_{t}} c_{t}^{i}-\sum_{i=1}^{w_{t}-1} c_{t}^{i} t_{k-w_{t}+i}\right), 0.2 T_{\alpha}\right\}
$$

such that:

$$
t_{k-w_{q}+i}<T_{A d}^{2}\left[k-w_{t}+i\right] \text { for } i=1,2, \ldots, w_{t}-1
$$

where $c$ and $w$. are respectively the exponential adaptation factor and the adaptation window length for the associated threshold. And the WTS is considered faulty whenever one of the $Q$ and $T^{2}$ statistics exceeds its instantaneous threshold.

These adaptive thresholds, given by equations (13 to 16), consist of a fixed part that controls the deviation from the preset fixed limits and a dynamic part on the basis of a modified EWMA control chart. The exponential filtering increases the reliability and robustness against data outliers and thus it greatly reduces false alarms in the FD system. Besides, this method keeps the core information carried in each statistic since it gives greater importance to recent data while still considering previous values with the most recent value having the highest weight and thus it correctly ensures fast detection of small and abrupt abnormalities with a high detection sensitivity. Moreover, associated conditions for these thresholds make the adaptation based on the normal operation statistics only, so additionally, the thresholds measure the deviation of the WTS from previous normal operation mode.

\section{Application setup}

\begin{tabular}{|c|c|c|c|}
\hline Scenario & Type & Scenario & Description \\
\hline 1 & 1a) & Pitch angle sensor, stuck & A fixed value of $\beta_{1, m 1}$ equal to $5^{\circ}$ \\
\hline 2 & 1b) & Pitch angle sensor, scale & A gain factor on $\beta_{2, m 2}$ of 3.2 \\
\hline 3 & 4a) & Torque offset & An offset on $\tau_{g}$ \\
\hline 4 & 2a) & Speed sensor, stuck & A constant value of $\omega_{r, m 1}$ equal to $1.4 \mathrm{rad} / \mathrm{s}$ \\
\hline 5 & $\begin{array}{l}2 b), \\
3 b)\end{array}$ & Speed sensors, scale & A gain factor on $\omega_{r, m 2}$ and $\omega_{g, m 1}$ of 1.2 and 0.8 , respectively \\
\hline 6 & 5a) & Slow control & $\begin{array}{l}\text { Change in the dynamics }\left(\xi, \omega_{n}\right) \text { due to the hydraulic pressure drop of pitch } \\
\text { actuator } 2\end{array}$ \\
\hline 7 & $5 b)$ & Slow control & $\begin{array}{l}\text { Change in the dynamics }\left(\xi, \omega_{n}\right) \text { due to increased air content in the oil on } \\
\text { pitch actuator } 2\end{array}$ \\
\hline 8 & 1a) & Pitch angle sensor, stuck & A fixed value of $\beta_{3, m 1}$ equal to $10^{\circ}$ \\
\hline 9 & 6 & Drive train & Change in the friction in the drive train \\
\hline 10 & 3a) & Generator speed sensor, stuck & A fixed value of $\omega_{g, m 1}$ equal to $70 \mathrm{rad} / \mathrm{s}$ \\
\hline
\end{tabular}

Table 3. Fault scenarios applied in the wind turbine benchmark model [37, 38, 39].

Using a real measured wind speed, the system is studied in both functional zones, and static as well as dynamic monitoring tasks are performed. In this direction, PCA models are constructed for both operation modes where the switching between them is carried out following the system control, based on input mean wind speed and output generated power. Among the signals indicated in Table 1, which are in general used for FD, only measured signals are used in our application to avoid extra hardware redundancy. Namely the duplicated measurements of the three pitch positions $\beta_{i, m j}$ as well as the generator and the rotor speeds $\omega_{g, m j}$ and $\omega_{r, m j}$ respectively $(i=1,2,3$, and $j=1,2)$, in addition to the single measurements of wind speed $v_{w, m}$, generator torque $\tau_{g, m}$, and generated power $P_{g, m}$ are retained. This results in 13 measured variables, indicated in Fig. 3 and well described in section 2, which are used here to train a statistical model for the wind turbine system and used for FD analysis and performance evaluation. Thus each measurement dataset $\boldsymbol{X}$ is constructed in this form: 


$$
\boldsymbol{X}_{\mathbf{0}}=\left[v_{w, m}, \omega_{r, m 1}, \omega_{r, m 2}, \omega_{g, m 1}, \omega_{g, m 2}, \tau_{g, m}, P_{g, m}, \beta_{1, m 1}, \beta_{1, m 2}, \beta_{2, m 1}, \beta_{2, m 2}, \beta_{3, m 1}, \beta_{3, m 2}\right]
$$

Different datasets are acquired for statistical learning, model validation, as well as test and evaluation. For each operation zone, both static and dynamic PCA models are trained through 500 samples collected within 500 seconds (with one second sampling time) during normal system operation under the associated mode, $\boldsymbol{X}_{\mathbf{0}} \in \boldsymbol{R}^{500 \times 13}$. The overall adaptive threshold schemes are also tuned using training datasets along with model parameters. Each constructed scheme is then tested and validated online through a testing dataset of 100000 samples collected with 10 ms sampling time and spanning 1000 seconds operation period, $\boldsymbol{X}_{\mathbf{0}} \in \boldsymbol{R}^{100,000 \times 13}$. The difference in duration and sampling time of the training and validation datasets ensure both application generality and independence of the monitoring performance from training data.

The robustness of each scheme to outliers caused by the measurement devices noise and inaccuracies is first checked online through normal operation testing datasets. False Alarms Rate (FAR) performance index is used in this purpose to assess the robustness of the scheme; the FAR index is defined as the rate of normal operation samples which are falsely identified as faults $N_{f}$ over the total number of evaluated samples during normal operation $N_{n}$.

$$
F A R=100 \frac{N_{f}}{N_{n}} \%
$$

The constructed schemes are then evaluated for online fault detection. The 10 fault scenarios described in Table 3 are independently and successively simulated in different operation zones. Each faulty set is simulated over a 200 seconds operation period and consists of 20000 samples; one fault scenario is introduced in each set after 50 seconds of normal operation, thus each faulty set consists of 5000 normal operation samples followed by 15000 faulty samples, $\boldsymbol{X}_{\mathbf{0}} \in \boldsymbol{R}^{20,000 \times 13}$. In this case, the fault Detection time Delay (DD) is used to assess the FD performance, it represents the time delay between the detection and real occurrence of the fault.

$$
D D=\text { detection time - occurrence time }
$$

The resulting schemes are compared in terms of robustness and FD performance. A more robust scheme has the lowest FAR since any false alarm may cause an unnecessary shut down of the system and thus reduces the monitoring reliability of the scheme. At the same time, the objective of an effective FD scheme is to detect possible system faults as fast as possible with minimum delay to prevent the fault from evolving and causing overall system failure as well as to ensure sufficient time to do necessary counteractions. Thus the overall performance is assessed by achieving the minimum FAR and lowest DD to ensure respectively robustness and efficiency.

\section{Results and discussion}

Data from WTSs form two particular and challenging backgrounds for analysis; the noisy environment due to measurements acquisition through electromechanical sensors, in addition to recurrent transient phases. These properties result in a non-stationary and unsteady process. Under such particular conditions, the representation and estimation of signals patterns are challenging. Moreover, the switching control strategy of the WTS and its fast dynamics both contribute to this challenge leading to continuously chattering modes. Discriminating changes due to anomalies from those due to evolution of normal operation and sparse changes is not an easy task. In this direction, figure 5 illustrates the main physical input and generated output over just 2 hours of normal operation. The real measured sequence of wind speed driving the system has a considerable signal-to-noise-ratio and changing trends forcing the system to switch operation and undergo many transient phases. This can be seen through the output generated power with fluctuating amplitude as well as a significant level of measurement noise. Notice that the operation modes of the system are continuously switching. The data in its whole range, of just these two variables, do not follow a particular pattern. Furthermore, substantial changes may evolve over longer operation periods; System functionality in its whole range and signals maximum variations must therefore be well considered in both statistical modelling and evaluation. 


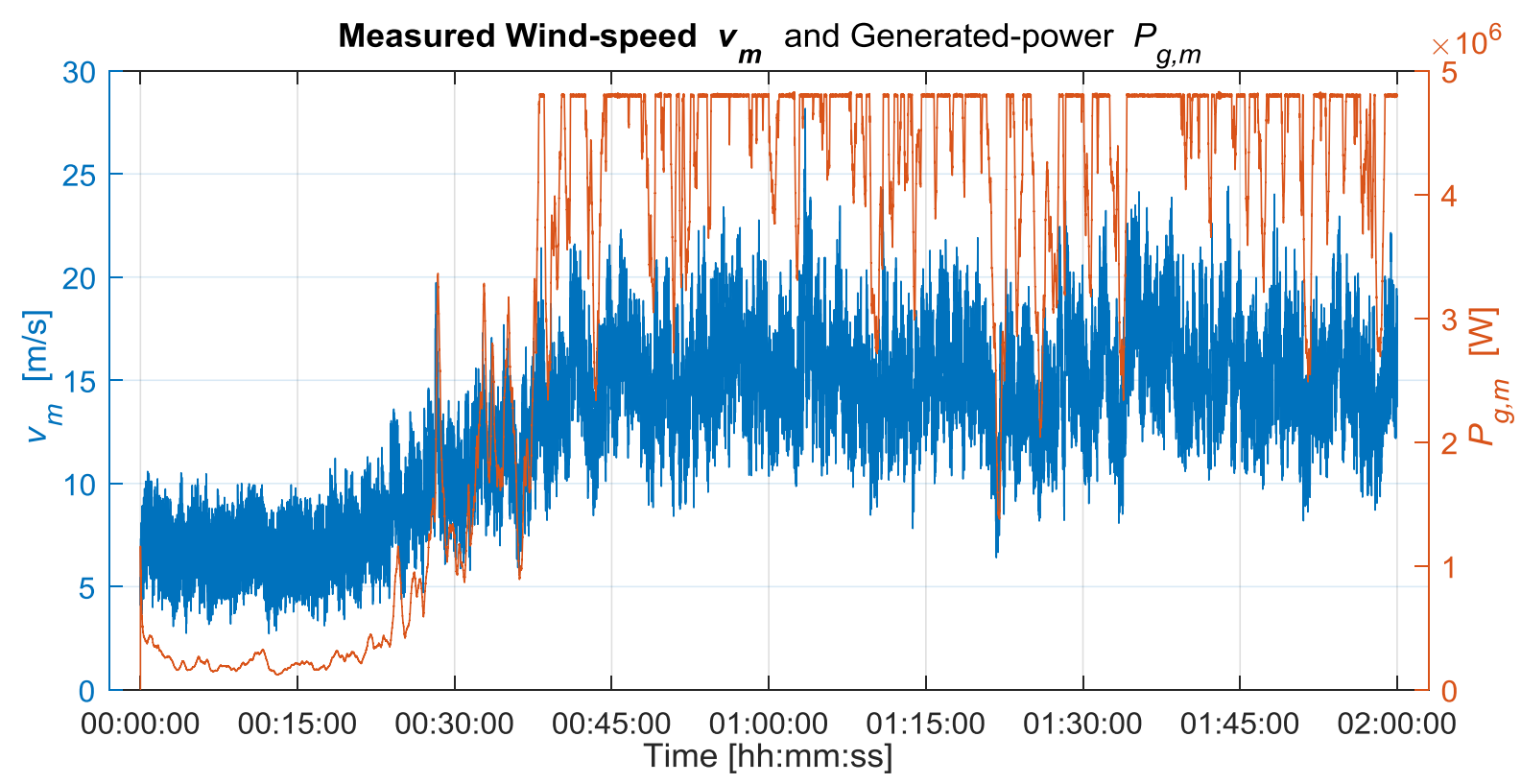

Fig 5. Trends and variations of the input wind speed and the output generated power over 2 hours of WTS operation under normal conditions.

Such particular conditions in WTS pose hard challenges for the design of a reliable FD approach in its offline training stage and online detection. Training an efficient PCA model generally applies to linear processes with data approaching a multivariate Gaussian distribution which is clearly not the case for this system. For this reason, multiPCA-models are used to monitor the system under each operation zone. The training datasets from both zones are collected according to the controller action to ensure correct statistical modeling. Even though PCA is a powerful noise decoupling tool, the projection of new measurements on the trained models will still cause many outliers. A solution to this issue is the use of the adaptive threshold scheme based on a modified EWMA low-pass filter, validation of the detection algorithm and analysis of its robustness to outliers are provided below.

Table 4. False alarms contribution (lower is better) of different schemes during normal operation modes.

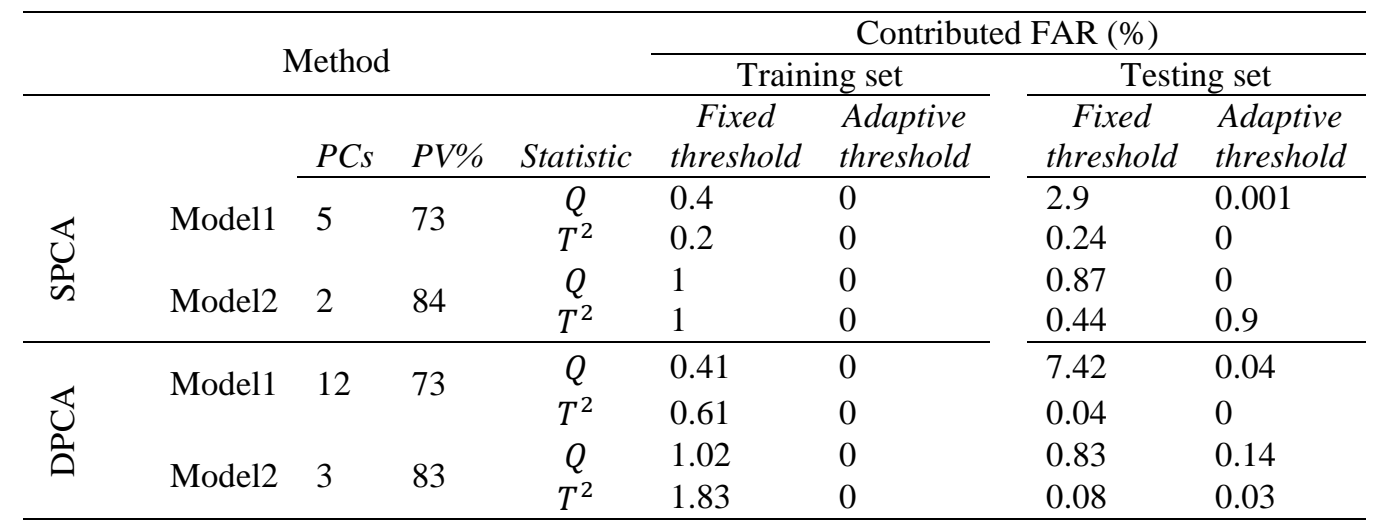

The recommended training datasets are acquired during normal conditions of the WTS under both functional zones, these datasets are then used to train parallel statistical models. Both static and dynamic PCA models are constructed using Kaiser's rule for retaining the appropriate number of principal components. Kaiser's rule is also used to conclude the number of nonzero eigenvalues and thus the number of linear relationships to determine the number of lags in DPCA method. As a result, DPCA models of zone II and zone III are constructed with two lags to capture serial correlation and model the system dynamics. The resulting parameters of each model such as the number of retained PCs and the captured percentage variance (PV\%) are listed in Table 4, where Model1 and Model2 respectively correspond to zone II and zone III for both static (SPCA) and dynamic (DPCA) cases. This table also lists the $Q$ and $T^{2}$ contributions of FAR (lower is better) of each model in both training and testing datasets. A conclusion can be drawn regarding the validity and applicability of the proposed framework, this ensures a stable performance as demonstrated with a tolerable FAR (type I error) in both training and testing steps with a FAR of $0 \%$ to $7.41 \%$. It is also noted that the integration of the proposed adaptive threshold scheme with each of the constructed models has a great effectiveness in eliminating/reducing the excessive numbers of false alarms contributed by the conventional fixed thresholds, and thus ensuring the FD robustness through both statistics with less than $1 \%$ false alarms. 
Scenario: 1

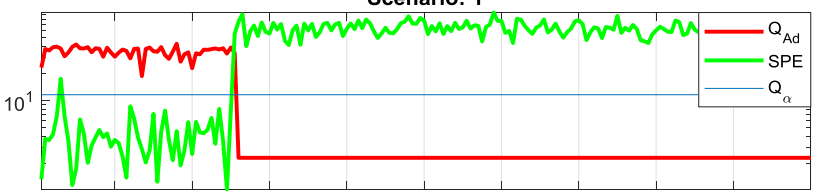

\begin{tabular}{lllllllllll} 
& 20 & 40 & 60 & 80 & 100 & 120 & 140 & 160 & 180 & 20 \\
\hline
\end{tabular}

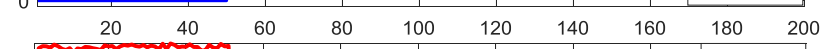

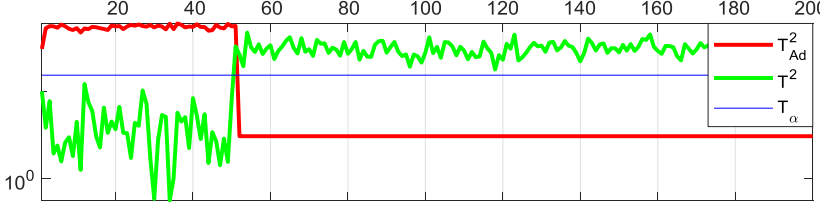

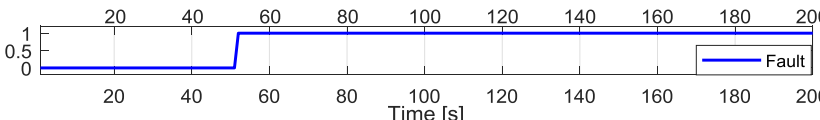

Scenario: 5

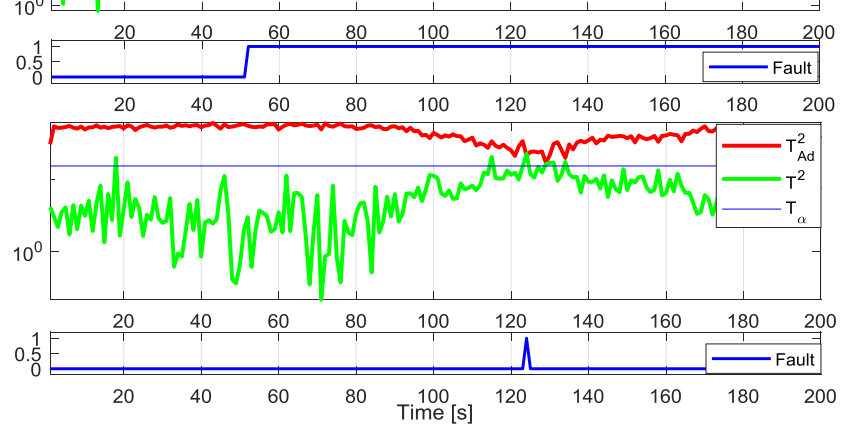

Fig 6. SPCA_AD Model_1 monitoring results during Zone_II operation mode, Scenario_1: fixed-value single sensor fault, and Scenario_5: gain-factor multiple sensors faults.

Scenario: 8
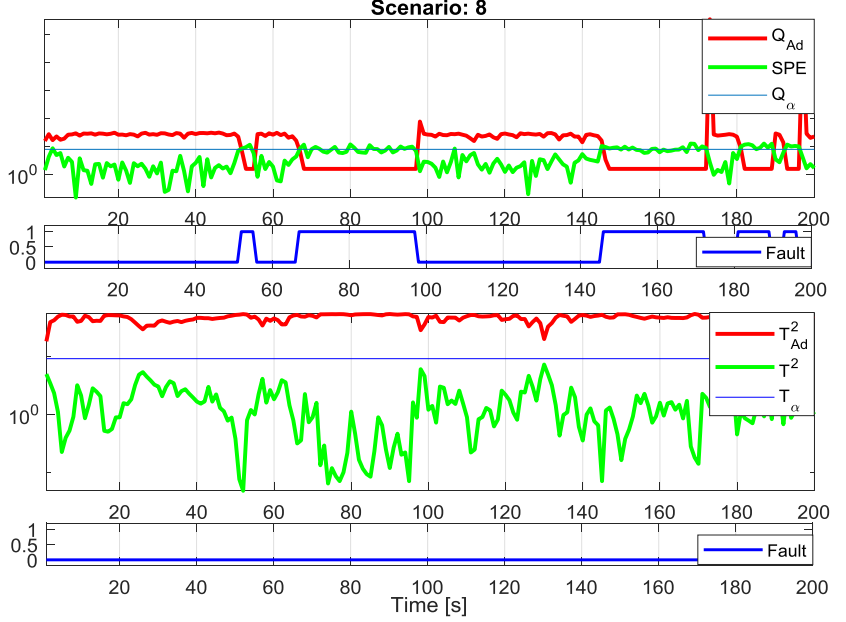

Scenario: 9
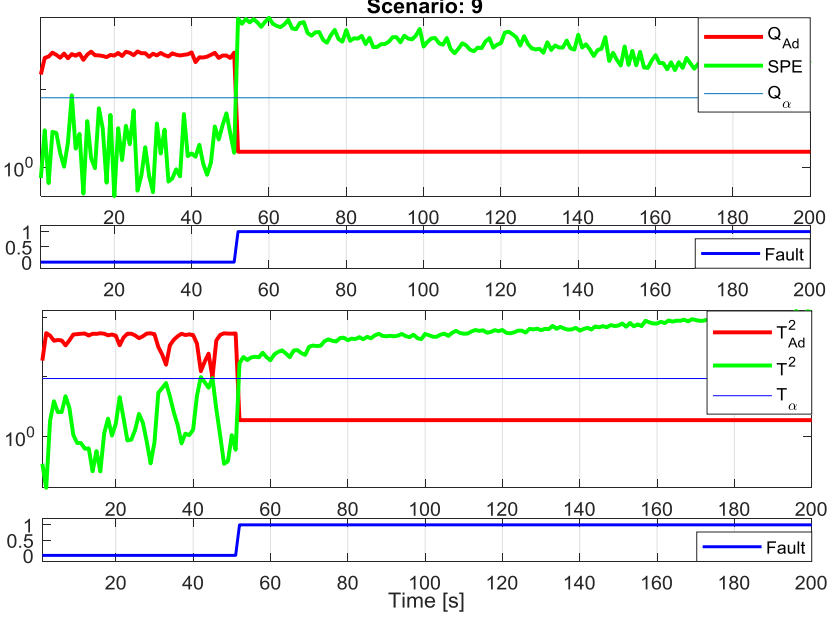

Fig 7.SPCA_AD MODEL_2 monitoring results during Zone_III operation mode, Scenario_8: sensor fault, and Scenario9: system fault.

After validation and robustness analysis, the constructed framework is tested for its online detection performance across all types of faults using both static and dynamic models for each operation zone. Monitoring results of both $Q$ and $T^{2}$ statistics are illustrated in Figures 6 to 9 across different faults; the resulting statistics are indicated in green with their fixed thresholds with blue and adaptive thresholds in red, also a fault indicator for the adaptive threshold is added to demonstrate the detection of a particular fault. These figures are plotted with a 1s sampling time for clarity purposes while the real sampling rate is $10 \mathrm{~ms}$. Recall that a fault is detected once a statistic exceeds its threshold, and that the faults are introduced after 50 seconds of normal operation.
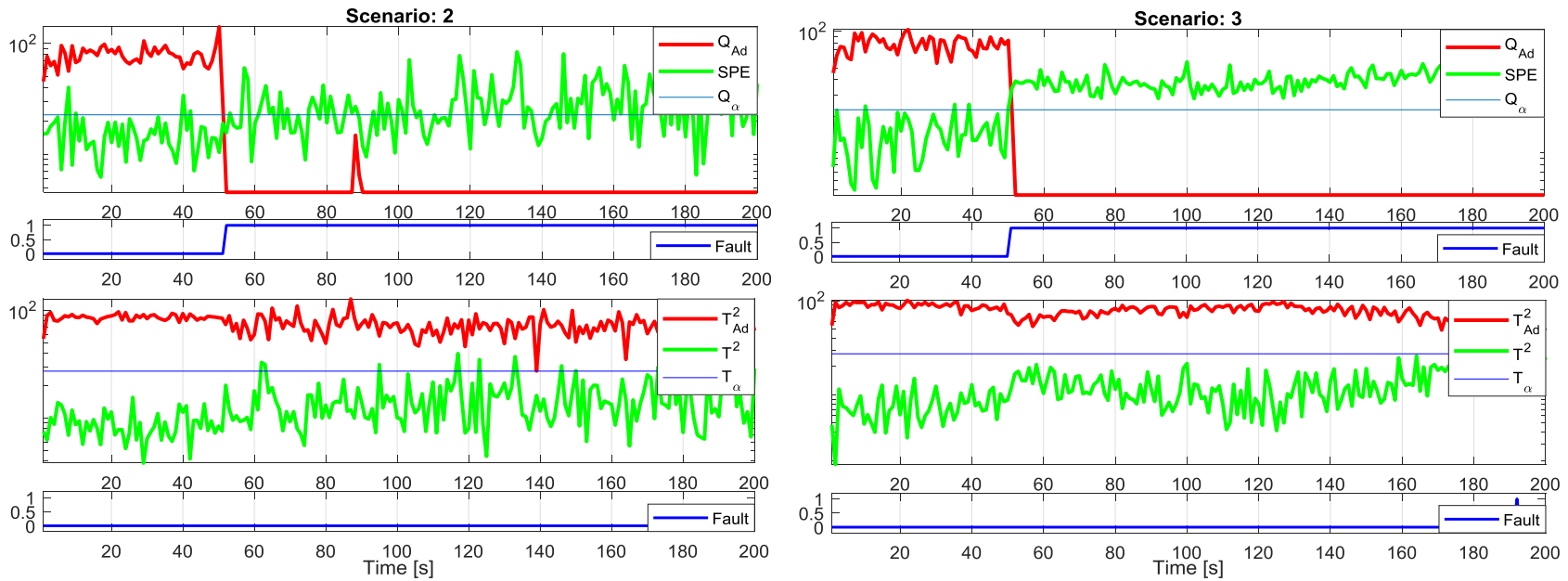
Fig 8. DPCA_AD MODEL_1 monitoring results during Zone_II operation mode, Scenario_2: sensor fault, and Scenario_3: offset actuator fault.

Figures 6 and 7 include results of static modelling of multi-PCA-models for zones II and III respectively, while figures 8 and 9 represent those of dynamic modelling. All figures demonstrate the successful detection of all faults, the detection time delay can be measured knowing the time of occurrence for each fault. These figures demonstrate the robustness of the proposed framework, this can be deduced from the number of false alarms during the first 50 seconds of normal behavior since no statistic is supposed to exceed its threshold. A comparison of fixed and adaptive thresholds shows that the later one is more robust. The detection sensitivity is also seen during faulty region through early fault detection and detection rate; each statistic here is supposed to exceed its threshold. These figures show that the $Q$ statistic has a better detection performance.
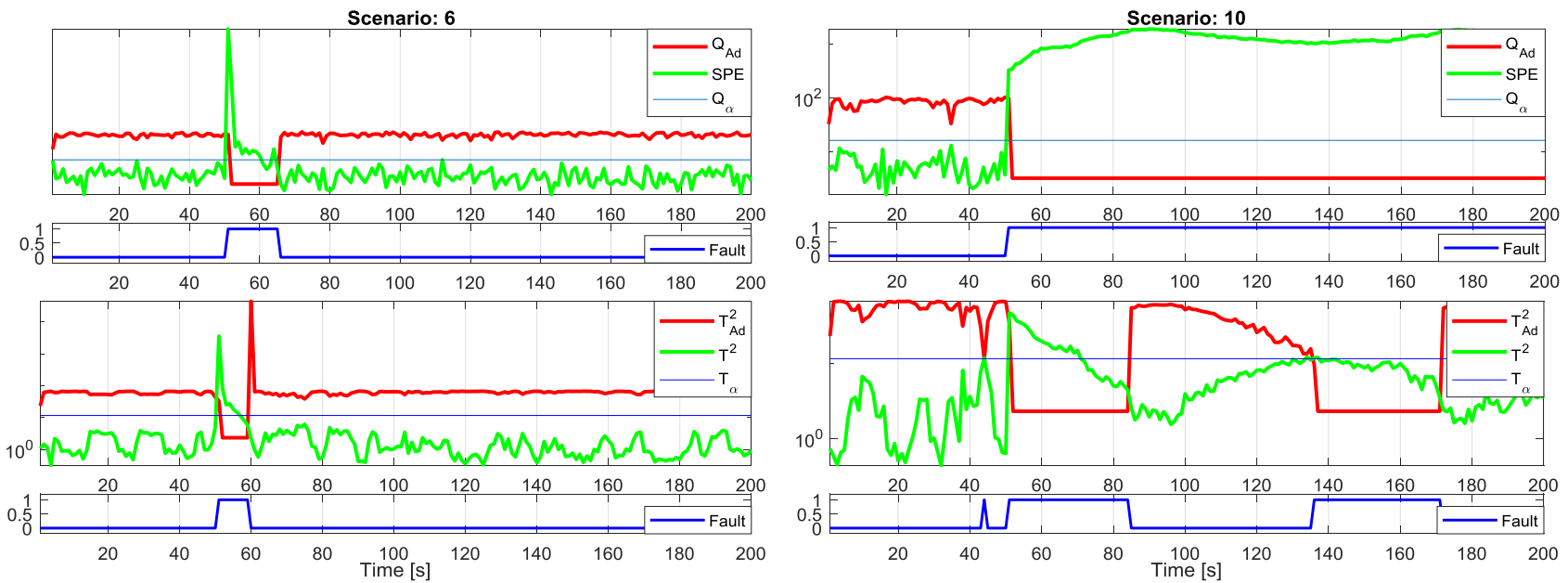

Fig 9. DPCA_AD MODEL_2 monitoring results during Zone_III operation mode, Scenario_6: Actuator fault, and Scenario_10: sensor fault.

Table 5. Fault detection performance results and comparisons across benchmark fault scenarios.

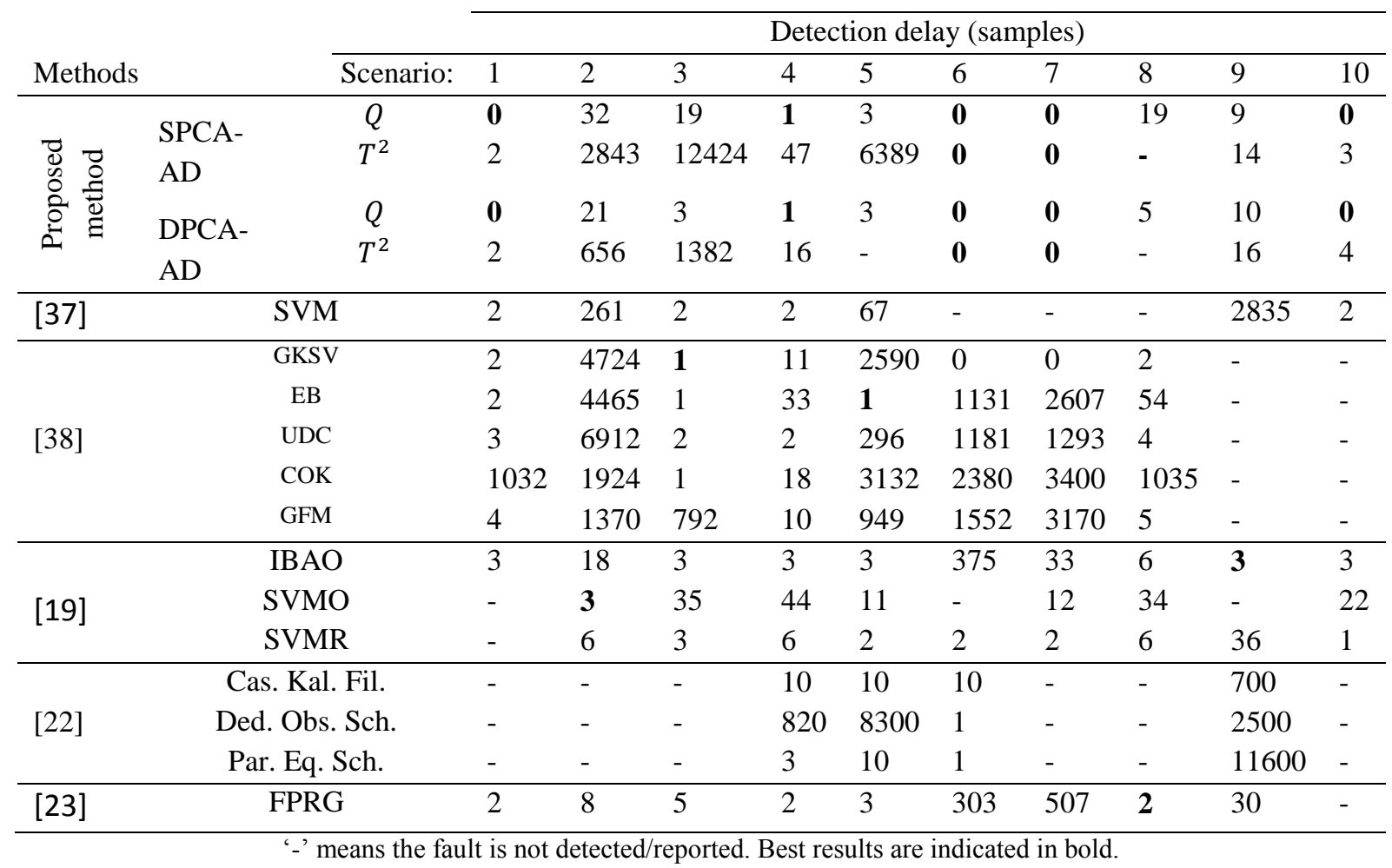

The obtained results are also summarized in Table 5, which lists the detection performance of static and dynamic PCA-based schemes across the different scenarios as previously described in Table 3 . Since the use of fixed control limits contributes excessive numbers of false alarms making the detection indefinite, Table 5 lists only those results obtained through adaptive thresholds. The results indicate that the $Q$ statistic has a better detection performance 
compared to the $T^{2}$ statistic as it detects the faults earlier; similarly, including the system dynamics through DPCA-AD method has shown better detection performance. Table 5 also includes comparisons with results reported by [19, 22, 23, 37, 38]. Some faults are detected instantaneously (with zero DD). Some scenarios corresponding to faults in sensors are detected with larger detection delays, such as the second scenario where the fault is detected after 21 faulty samples. Recall that sensor faults have a low severity as mentioned before, such a detection delay is acceptable. The rest of the faults are detected with very small detection delay, and they are within the detection time constraints as listed in Table 2 above. In addition to the fact that the proposed FD framework is semi-supervised and independent of its training data, the obtained DD results across the different benchmark faults prove the effectiveness and reliability of the developed schemes.

\section{Conclusions}

In this paper, a novel data-driven framework is presented for online fault detection in modern wind turbine systems. A minimum set of fault-relevant monitoring variables is recommended to achieve better performance and avoid redundancy of sensory devices. Informative datasets are therefore constructed to statistically model the system functionality under a highly noisy environment and chattering modes. Parallel multivariate statistical models are hence suggested to deal with the multi-model operations of the WTS and its switching control strategy as well as recurrent transient stages due to its fast dynamics. One presented algorithm incorporates static and dynamic PCA methods for data dimensionality reduction and parallel statistical modelling, and it is reinforced through a modified EWMA-based adaptive threshold for proper evaluation of the resulting statistics and the operation status. Moreover, a case study on a 4.8 MW benchmark was carried out analyzing the detection/detectability of common faults in sensors and actuators as well as system faults. The study included both functional zones during maximum and constant rated power generation depending on the available wind speed. The benchmark reflects real-time measurements under realistic conditions and it is used as a source of data to design the proposed strategy which can be easily generalized to practical applications. The proposed FD framework forms an effective semi-supervised approach which is independent of both faulty information and training data during offline learning and online FD stages, respectively. Compared to other methods in the literature, it is worth to mention that this algorithm shows superior results in terms of robustness to outliers and high detection sensitivity. Another remarkable advantage of the proposed method is its higher potential applications since it can be generalized to wind turbine systems of different sizes and control structures.

\section{References}

[1] https://windeurope.org/about-wind/, /statistics/, /reports/. Accessed December 2017.

[2] http://www.wind-energy-the-facts.org. Accessed December 2017.

[3] Tavner PJ. How are we going to make offshore wind farms more reliable? Present. SUPERGEN Wind 2011 Gen. Assem., Durham; 2011.

[4] Gustavo de Novaes Pires Leite, Alex Maurício Araújo, Pedro André Carvalho Rosas, Prognostic techniques applied to maintenance of wind turbines: a concise and specific review, In Renewable and Sustainable Energy Reviews, Volume 81, Part 2, 2018, Pages 1917-1925, ISSN 1364-0321, https://doi.org/10.1016/j.rser.2017.06.002.

[5] http://www.bbc.com/news/av/uk-16104630/40000-in-scotland-still-without-power-after-storms, Accessed December 2017.

[6] http://epaw.org/photos/WKA_ausgebrannt_5.jpg, Accessed December 2017.

[7] Raúl Ruiz de la Hermosa González-Carrato, Fausto Pedro García Márquez, Vichaar Dimlaye, Maintenance management of wind turbines structures via MFCs and wavelet transforms, In Renewable and Sustainable Energy Reviews, Volume 48, 2015, Pages 472-482, ISSN 1364-0321, https://doi.org/10.1016/j.rser.2015.04.007.

[8] Rashad M. Kamel, Three fault ride through controllers for wind systems running in isolated micro-grid and Effects of fault type on their performance: A review and comparative study, In Renewable and Sustainable Energy Reviews, Volume 37, 2014, Pages 698-714, ISSN 1364-0321, https://doi.org/10.1016/j.rser.2014.05.065.

[9] Jackson John Justo, Francis Mwasilu, Jin-Woo Jung, Doubly-fed induction generator based wind turbines: A comprehensive review of fault ride-through strategies, In Renewable and Sustainable Energy Reviews, Volume 45, 2015, Pages 447-467, ISSN 1364-0321, https://doi.org/10.1016/j.rser.2015.01.064.

[10] Yonggang Lin, Le Tu, Hongwei Liu, Wei Li, Fault analysis of wind turbines in China, In Renewable and Sustainable Energy Reviews, Volume 55, 2016, Pages 482-490, ISSN 1364-0321, https://doi.org/10.1016/j.rser.2015.10.149.

[11] Aubryn Cooperman, Marcias Martinez, Load monitoring for active control of wind turbines, In Renewable and Sustainable Energy Reviews, Volume 41, 2015, Pages 189-201, ISSN 1364-0321, https://doi.org/10.1016/j.rser.2014.08.029.

[12] Mathew L. Wymore, Jeremy E. Van Dam, Halil Ceylan, Daji Qiao, A survey of health monitoring systems for wind turbines, In Renewable and Sustainable Energy Reviews, Volume 52, 2015, Pages 976-990, ISSN 1364-0321, https://doi.org/10.1016/j.rser.2015.07.110. 
[13] A. Hamdan, F. Mustapha, K.A. Ahmad, A.S. Mohd Rafie, A review on the micro energy harvester in Structural Health Monitoring (SHM) of biocomposite material for Vertical Axis Wind Turbine (VAWT) system: A Malaysia perspective, In Renewable and Sustainable Energy Reviews, Volume 35, 2014, Pages 23-30, ISSN 1364-0321, https://doi.org/10.1016/j.rser.2014.03.050.

[14] W.Y. Liu, B.P. Tang, J.G. Han, X.N. Lu, N.N. Hu, Z.Z. He, The structure healthy condition monitoring and fault diagnosis methods in wind turbines: A review, In Renewable and Sustainable Energy Reviews, Volume 44, 2015, Pages 466-472, ISSN 1364-0321, https://doi.org/10.1016/j.rser.2014.12.005.

[15] Maria Martinez-Luengo, Athanasios Kolios, Lin Wang, Structural health monitoring of offshore wind turbines: A review through the Statistical Pattern Recognition Paradigm, In Renewable and Sustainable Energy Reviews, Volume 64, 2016, Pages 91-105, ISSN 1364-0321, https://doi.org/10.1016/j.rser.2016.05.085.

[16] Bin Yang, Dongbai Sun, Testing, inspecting and monitoring technologies for wind turbine blades: A survey, In Renewable and Sustainable Energy Reviews, Volume 22, 2013, Pages 515-526, ISSN 1364-0321, https://doi.org/10.1016/j.rser.2012.12.056.

[17] Henrique Dias Machado de Azevedo, Alex Maurício Araújo, Nadège Bouchonneau, A review of wind turbine bearing condition monitoring: State of the art and challenges, In Renewable and Sustainable Energy Reviews, Volume 56, 2016, Pages 368-379, ISSN 1364-0321, https://doi.org/10.1016/j.rser.2015.11.032.

[18]Zhimin Yang, Yi Chai, A survey of fault diagnosis for onshore grid-connected converter in wind energy conversion systems, In Renewable and Sustainable Energy Reviews, Volume 66, 2016, Pages 345-359, ISSN 1364-0321, https://doi.org/10.1016/j.rser.2016.08.006.

[19]H. Sanchez, T. Escobet, V. Puig and P. F. Odgaard, "Fault Diagnosis of an Advanced Wind Turbine Benchmark Using Interval-Based ARRs and Observers," in IEEE Transactions on Industrial Electronics, vol. 62, no. 6, pp. 3783-3793, June 2015. doi: 10.1109/TIE.2015.2399401

[20] Rosa M. Fernandez-Canti, Joaquim Blesa, Sebastian Tornil-Sin, Vicenç Puig, Fault detection and isolation for a wind turbine benchmark using a mixed Bayesian/Set-membership approach, In Annual Reviews in Control, Volume 40, 2015, Pages 59-69, ISSN 1367-5788, https://doi.org/10.1016/j.arcontrol.2015.08.002.

[21] X. Liu, Z. Gao and M. Z. Q. Chen, "Takagi-Sugeno Fuzzy Model Based Fault Estimation and Signal Compensation With Application to Wind Turbines," in IEEE Transactions on Industrial Electronics, vol. 64, no. 7, pp. 5678-5689, July 2017. doi: 10.1109/TIE.2017.2677327

[22] S. Dey, P. Pisu and B. Ayalew, "A Comparative Study of Three Fault Diagnosis Schemes for Wind Turbines," in IEEE Transactions on Control Systems Technology, vol. 23, no. 5, pp. 1853-1868, Sept. 2015. doi: 10.1109/TCST.2015.2389713

[23] S. Simani, S. Farsoni and P. Castaldi, "Fault Diagnosis of a Wind Turbine Benchmark via Identified Fuzzy Models," in IEEE Transactions on Industrial Electronics, vol. 62, no. 6, pp. 3775-3782, June 2015. doi: 10.1109/TIE.2014.2364548

[24] Silvio Simani, Paolo Castaldi, Data-driven and adaptive control applications to a wind turbine benchmark model, In Control Engineering Practice, Volume 21, Issue 12, 2013, Pages 1678-1693, ISSN 0967-0661, https://doi.org/10.1016/j.conengprac.2013.08.009.

[25] Iury Valente de Bessa, Reinaldo Martinez Palhares, Marcos Flávio Silveira Vasconcelos D'Angelo, João Edgar Chaves Filho, Data-driven fault detection and isolation scheme for a wind turbine benchmark, In Renewable Energy, Volume 87, Part 1, 2016, Pages 634-645, ISSN 0960-1481, https://doi.org/10.1016/j.renene.2015.10.061.

[26] G.Wang, J. Liu, Y. Zhang, Y. Li (2015), A novel multi-mode data processing method and its application in industrial process monitoring, 29, pages 126-138, J. Chemometrics, doi: 10.1002/cem.2686.

[27] Yingwei Zhang, Jiayu An, Hailong Zhang, Monitoring of time-varying processes using kernel independent component analysis, In Chemical Engineering Science, Volume 88, 2013, Pages 23-32, ISSN 0009-2509, https://doi.org/10.1016/j.ces.2012.11.008.

[28] Qian Kang, Qingguo Ru, Yan Liu, Lingyan Xu, Jia Liu, Yifei Wang, Yewen Zhang, Hui Li, Qing Zhang, Qing Wu, On-line monitoring the extract process of Fu-fang Shuanghua oral solution using near infrared spectroscopy and different PLS algorithms, In Spectrochimica Acta Part A: Molecular and Biomolecular Spectroscopy, Volume 152, 2016, Pages 431-437, ISSN 1386-1425, https://doi.org/10.1016/j.saa.2015.07.098.

[29] G. Jia, Y. Wang, B. Huang. Dynamic higher-order cumulants analysis for state monitoring based on a novel lag selection. Information Sciences. 2016;331:45-66.

[30] Jiawei Tang, Xuefeng Yan, Neural network modeling relationship between inputs and state mapping plane obtained by FDA-t-SNE for visual industrial process monitoring, In Applied Soft Computing, Volume 60, 2017, Pages 577590, ISSN 1568-4946, https://doi.org/10.1016/j.asoc.2017.07.022.

[31] Feihong Guo, Chao Shang, Biao Huang, Kangcheng Wang, Fan Yang, Dexian Huang, Monitoring of operating point and process dynamics via probabilistic slow feature analysis, In Chemometrics and Intelligent Laboratory Systems, Volume 151, 2016, Pages 115-125, ISSN 0169-7439, https://doi.org/10.1016/j.chemolab.2015.12.017. 
[32] Azzeddine Bakdi, Abdelmalek Kouadri, Abderazak Bensmail, Fault detection and diagnosis in a cement rotary kiln using PCA with EWMA-based adaptive threshold monitoring scheme, In Control Engineering Practice, Volume 66, 2017, Pages 64-75, ISSN 0967-0661, https://doi.org/10.1016/j.conengprac.2017.06.003.

[33] Azzeddine Bakdi, Abdelmalek Kouadri, A new adaptive PCA based thresholding scheme for fault detection in complex systems, In Chemometrics and Intelligent Laboratory Systems, Volume 162, 2017, Pages 83-93, ISSN 0169-7439, https://doi.org/10.1016/j.chemolab.2017.01.013.

[34] Tiago J. Rato, Marco S. Reis, Defining the structure of DPCA models and its impact on process monitoring and prediction activities, In Chemometrics and Intelligent Laboratory Systems, Volume 125, 2013, Pages 74-86, ISSN 0169-7439, https://doi.org/10.1016/j.chemolab.2013.03.009.

[35] W. Ku, R. H. Storer, C. Georgakis. Disturbance detection and isolation by dynamic principal component analysis. Chemometrics and Intelligent Laboratory Systems. 1995;30:179-196.

[36] Lou, Z., Shen, D. and Wang, Y. (2017), Two-step principal component analysis for dynamic processes monitoring. Can. J. Chem. Eng.. doi:10.1002/cjce.22855.

[37] NassimLaouti, NidaSheibat-Othman, Sami Othman, Support Vector Machines for Fault Detection in Wind Turbines, In IFAC Proceedings Volumes, Volume 44, Issue 1, 2011, Pages 7067-7072, ISSN 1474-6670, ISBN 9783902661937, https://doi.org/10.3182/20110828-6-IT-1002.02560.

[38]P. F. Odgaard, J. Stoustrupand M. Kinnaert, "Fault-Tolerant Control of Wind Turbines: A Benchmark Model," in IEEE Transactions on Control Systems Technology, vol. 21, no. 4, pp. 1168-1182, July 2013. doi: 10.1109/TCST.2013.2259235

[39] P. F. Odgaard and J. Stoustrup, "A Benchmark Evaluation of Fault Tolerant Wind Turbine Control Concepts," in IEEE Transactions on Control Systems Technology, vol. 23, no. 3, pp. 1221-1228, May 2015. doi: 10.1109/TCST.2014.2361291

[40] S. Wei, L. Zhang, Y. Xu, Y. Fu and F. Li, "Hierarchical Optimization for the Double-Sided Ring Structure of the Collector System Planning of Large Offshore Wind Farms," in IEEE Transactions on Sustainable Energy, vol. 8, no. 3, pp. 1029-1039, July 2017. doi: 10.1109/TSTE.2016.2646061

[41] H. Badihi, Y. Zhang and H. Hong, "Wind Turbine Fault Diagnosis and Fault-Tolerant Torque Load Control Against Actuator Faults," in IEEE Transactions on Control Systems Technology, vol. 23, no. 4, pp. 1351-1372, July 2015. doi: 10.1109/TCST.2014.2364956 